TRANSACTIONS OF THE

AMERICAN MATHEMATICAL SOCIETY

Volume 356, Number 6, Pages 2459-2480

S 0002-9947(03)03374- 9

Article electronically published on November 25, 2003

\title{
NORMS OF LINEAR-FRACTIONAL COMPOSITION OPERATORS
}

\author{
P. S. BOURDON, E. E. FRY, C. HAMMOND, AND C. H. SPOFFORD
}

\begin{abstract}
We obtain a representation for the norm of the composition operator $C_{\phi}$ on the Hardy space $H^{2}$ whenever $\phi$ is a linear-fractional mapping of the form $\phi(z)=b /(c z+d)$. The representation shows that, for such mappings $\phi$, the norm of $C_{\phi}$ always exceeds the essential norm of $C_{\phi}$. Moreover, it shows that a formula obtained by Cowen for the norms of composition operators induced by mappings of the form $\phi(z)=s z+t$ has no natural generalization that would yield the norms of all linear-fractional composition operators. For rational numbers $s$ and $t$, Cowen's formula yields an algebraic number as the norm; we show, e.g., that the norm of $C_{1 /(2-z)}$ is a transcendental number. Our principal results are based on a process that allows us to associate with each non-compact linear-fractional composition operator $C_{\phi}$, for which $\left\|C_{\phi}\right\|>\left\|C_{\phi}\right\|_{e}$, an equation whose maximum (real) solution is $\left\|C_{\phi}\right\|^{2}$. Our work answers a number of questions in the literature; for example, we settle an issue raised by Cowen and MacCluer concerning co-hyponormality of a certain family of composition operators.
\end{abstract}

\section{INTRODUCTION}

Let $\mathbb{U}$ denote the open unit disk in the complex plane and let $H^{2}$ denote the classical Hardy space of $\mathbb{U}$. Whenever $\phi$ is analytic on $\mathbb{U}$ with $\phi(\mathbb{U}) \subseteq \mathbb{U}$, the composition operator $C_{\phi}$, defined for $f \in H^{2}$ by $C_{\phi} f=f \circ \phi$, is bounded on $H^{2}$ (see, e.g., [10] or [21]). In 1988, Carl Cowen [8] obtained a formula for the precise norm of $C_{\phi}$ when $\phi(z)=s z+t$ for some complex constants $s$ and $t$ satisfying $|s|+|t| \leq 1$ :

$$
\left\|C_{\phi}\right\|=\sqrt{\frac{2}{1+|s|^{2}-|t|^{2}+\sqrt{\left(1-|s|^{2}+|t|^{2}\right)^{2}-4|t|^{2}}}} .
$$

Cowen's result, together with questions about composition-operator norms raised in [10, p. 125] and [11, has inspired a number of papers ([1, [2], 5], 14]).

In 1], Appel, Bourdon, and Thrall prove that the norm of $C_{\phi}$ cannot, in general, be computed based on the action of $C_{\phi}$ (or $C_{\phi}^{*}$ ) on Hardy-space reproducing kernels, answering a question raised in [10, p. 125]. In [14, Hammond obtains exact values for the norms of certain linear-fractional composition operators based on finite iteration of a functional equation satisfied by eigenvectors for $C_{\phi}^{*} C_{\phi}$. Here, based

Received by the editors September 4, 2002 and, in revised form, April 27, 2003.

2000 Mathematics Subject Classification. Primary 47B33.

This research was supported in part by a grant from the National Science Foundation (DMS0100290). 
on an infinite iterative process, we use Hammond's functional equation to represent the norm of any composition operator induced by a mapping of the form

$$
\phi(z)=b /(c z+d) \text { where }|d|-|c| \geq|b|,
$$

the inequality simply being the condition that guarantees that $\phi$ map $\mathbb{U}$ into itself.

Our work shows that, for $\phi$ satisfying (1.2), the norm of $C_{\phi}$ always exceeds the essential norm of $C_{\phi}$. It also shows that the norm of $C_{1 /(2-z)}$ is a transcendental number, from which it follows that there is no formula like (1.1) yielding the norms of all linear-fractional composition operators. We remark that our representation of the norm of $C_{2 /(3-z)}$ answers a question raised by Appel, Bourdon, and Thrall [1, Question 4.5].

Our most interesting results are consequences of Theorem 3.5 below, which identifies $\left\|C_{\phi}\right\|^{2}$ as the maximum solution of an auxiliary equation we associate with $\phi$, given that $C_{\phi}$ is a non-compact linear-fractional composition operator whose norm exceeds its essential norm. The proof of Theorem 3.5 yields a test (Corollary 3.6) for determining when the norm of $C_{\phi}$ exceeds its essential norm $\left\|C_{\phi}\right\|_{e}$. Using this test, we analyze the norm versus essential norm issue for composition operators induced by members of the Cowen-Kriete family:

$$
\phi_{r, s}(z)=\frac{(r+s) z+1-s}{r(1-s) z+1+s r},
$$

where $0<s<1$ and $-1<r \leq 1$.

Suppose that $0<s<1$. It follows from Cowen and Kriete's work in [9] that the operator $C_{\phi_{r, s}}^{*}$ is subnormal if and only if $0 \leq r \leq 1$. Thus, for $0 \leq r \leq 1$, the spectral radius of $C_{\phi_{r, s}}$ equals $\left\|C_{\phi_{r, s}}\right\|$. For composition operators induced by $\phi_{r, s}$, the spectral radius equals the essential norm (see the next section for a discussion); hence, for $0 \leq r \leq 1$, members of the Cowen-Kriete family induce composition operators with norm equaling essential norm. On the other hand, a consequence of Proposition 2.3.2 of [19] is that $\left\|C_{\phi_{r, s}}\right\|>\left\|C_{\phi_{r, s}}\right\|_{e}$ for $-1<r<-1 / 3$. We complete the story for real values of $r$, proving that $\left\|C_{\phi_{r, s}}\right\|>\left\|C_{\phi_{r, s}}\right\|_{e}$ whenever $-1<r<0$ (see Theorem 3.7 below). Our work shows that $\left\|C_{\phi_{r, s}}\right\|$ exceeds the spectral radius of $C_{\phi_{r, s}}$ when $r$ is negative; thus no member of the family $\phi_{r, s}$ with $-1<r<0$ can induce a co-hyponormal composition operator. This improves Corollary 1 of 12 and settles an issue raised in [11, p. 21].

If $C_{\phi}$ is compact, then of course $\left\|C_{\phi}\right\|_{e}=0$, so that $\left\|C_{\phi}\right\|$ always exceeds $\left\|C_{\phi}\right\|_{e}$ in this situation. Our results for the norms of compact composition operators with linear-fractional symbol are discussed in the final section of the paper. The CowenKriete family plays a role here as well.

\section{BACKGROUND}

The Hardy space $H^{2}$ is the Hilbert space consisting of analytic functions on $\mathbb{U}$ whose Taylor coefficients, in the expansion about the origin, are square summable. The inner product inducing the norm of $H^{2}$ is given by $\left\langle\sum_{n=0}^{\infty} a_{n} z^{n}, \sum_{n=0}^{\infty} b_{n} z^{n}\right\rangle=$ $\sum_{n=0}^{\infty} a_{n} \overline{b_{n}}$. The inner product of two functions $f$ and $g$ in $H^{2}$ may also be computed by integration:

$$
\langle f, g\rangle=\frac{1}{2 \pi} \int_{0}^{2 \pi} f\left(e^{i \theta}\right) \overline{g\left(e^{i \theta}\right)} d \theta,
$$

where $f$ and $g$ are defined a.e. on $\partial \mathbb{U}$ via radial limits (see, e.g., [13]). 
For $\alpha \in \mathbb{U}$, the reproducing kernel at $\alpha$ for $H^{2}$ is defined by

$$
K_{\alpha}(z)=\frac{1}{1-\bar{\alpha} z} .
$$

Easy computations show that $\left\langle f, K_{\alpha}\right\rangle=f(\alpha)$ whenever $f \in H^{2}$ and that $C_{\phi}^{*} K_{\alpha}=$ $K_{\phi(\alpha)}$, where $C_{\phi}^{*}$ denotes the adjoint of $C_{\phi}$. Using the reproducing property of $K_{\alpha}$ and the Cauchy-Schwarz inequality, one obtains the following estimate for all $\mathrm{H}^{2}$ functions $f$ :

$$
|f(\alpha)| \leq \frac{\|f\|_{H^{2}}}{\sqrt{1-|\alpha|^{2}}}, \alpha \in \mathbb{U} .
$$

In this paper, we are interested in linear-fractional composition operators on $H^{2}$, that is, composition operators on $H^{2}$ induced by linear-fractional mappings that take $\mathbb{U}$ into itself. Much is known about such composition operators; for example, spectral properties and cyclicity properties are completely understood (see, respectively, [10, Chapter 7] and [4]). These properties are largely determined by the fixed-point behavior of the inducing maps $\phi$.

Each analytic self-map $\phi$ of $\mathbb{U}$ that is not an elliptic automorphism of $\mathbb{U}$ has a unique attractive fixed point in the closure of $\mathbb{U}$, called the Denjoy-Wolff point of $\phi$. That is, if $\phi$ is not an elliptic automorphism, then there is a point $\omega$ in the closure of $\mathbb{U}$ - the Denjoy-Wolff point of $\phi$ - such that whenever $z \in \mathbb{U}$,

$$
\phi^{[n]}(z) \rightarrow \omega \quad \text { as } \quad n \rightarrow \infty,
$$

where $\phi^{[n]}$ denotes $\phi$ composed with itself $n$ times (and $\phi^{[0]}$ is the identity function). The Denjoy-Wolff point $\omega$ of $\phi$ may be characterized as follows:

- if $|\omega|<1$, then $\phi(\omega)=\omega$ and $\left|\phi^{\prime}(\omega)\right|<1$,

- if $|\omega|=1$, then $\phi(\omega)=\omega$ and $0<\phi^{\prime}(\omega) \leq 1$,

where, if $\omega \in \partial \mathbb{U}, \phi(\omega)$ is the angular limit of $\phi$ at $\omega$ and $\phi^{\prime}(\omega)$ is the angular derivative of $\phi$ at $\omega$.

Recall that $\phi$ is said to have angular derivative at $\zeta \in \partial \mathbb{U}$ if there is an $\eta \in \partial \mathbb{U}$ such that

$$
\angle \lim _{z \rightarrow \zeta} \frac{\phi(z)-\eta}{z-\zeta}
$$

is finite, where $\angle \lim$ denotes the angular (or non-tangential) limit. By the JuliaCarathéodory Theorem, $\phi$ has finite angular derivative at $\zeta$ if and only if $\phi^{\prime}$ has angular limit at $\zeta$ while $\phi$ has angular limit of modulus 1 at $\zeta$. For these results, the reader may consult [10, Chapter 2] or [21, Chapters 4 and 5].

One may use the Denjoy-Wolff point $\omega$ of $\phi$ and $\phi^{\prime}(\omega)$ to obtain information about $\left\|C_{\phi}\right\|$. The spectral radius of $C_{\phi}$, which we denote $\operatorname{rad}\left(C_{\phi}\right)$, is determined by $\omega$ as follows: if $\omega \in \mathbb{U}$, then $\operatorname{rad}\left(C_{\phi}\right)=1$; if $|\omega|=1$, then $\operatorname{rad}\left(C_{\phi}\right)=\left(\phi^{\prime}(\omega)\right)^{-1 / 2}($ [7] Theorem 2.1]). Thus, for example, $\left\|C_{\phi}\right\| \geq\left(\phi^{\prime}(\omega)\right)^{-1 / 2}$ whenever the Denjoy-Wolff point $\omega$ of $\phi$ lies on $\partial \mathbb{U}$.

If the Denjoy-Wolff point of $\phi$ happens to be the origin, then the following general estimate (see, e.g., [10, Corollary 3.7]) shows that $\left\|C_{\phi}\right\|=1$ :

$$
\frac{1}{1-|\phi(0)|^{2}} \leq\left\|C_{\phi}\right\|^{2} \leq \frac{1+|\phi(0)|}{1-|\phi(0)|} .
$$

However, the exact norm of $C_{\phi}$ is known in very few other situations. For inner functions (in particular the linear-fractional automorphisms of $\mathbb{U}$ ), Nordgren [15] 
showed that the square of the norm of $C_{\phi}$ is given by the rightmost quantity in (2.2); furthermore, Shapiro [20] showed that if $\phi$ is inner, then $\left\|C_{\phi}\right\|=\left\|C_{\phi}\right\|_{e}$. As we mentioned in the Introduction, Cowen obtained a formula for the norms of linearfractional composition operators induced by mappings of the form $\phi(z)=s z+t$. One of our goals is to represent the norm of a composition operator induced by a mapping of the form $\phi(z)=b /(c z+d)$.

Computing the essential norm of a composition operator is usually easier than computing its norm. For example, if $\phi$ is univalent (taking $\mathbb{U}$ to $\mathbb{U}$ ), Shapiro's essential norm formula 20 shows that

$$
\left\|C_{\phi}\right\|_{e}^{2}=\sup \left\{\frac{1}{\left|\phi^{\prime}(\zeta)\right|}: \zeta \in \partial \mathbb{U}\right\}
$$

where $\phi^{\prime}(\zeta)$ represents the angular derivative of $\phi$ at $\zeta$ (interpreted to be $\infty$ when $\phi^{\prime}(\zeta)$ does not exist). Because linear-fractional self-mappings of $\mathbb{U}$ are analytic on the closure of $\mathbb{U}$ (and, of course, univalent), one may compute the essential norm of $C_{\phi}$ when $\phi$ is linear fractional as

$$
\left\|C_{\phi}\right\|_{e}^{2}=\sup \left\{\frac{1}{\left|\phi^{\prime}(\zeta)\right|}: \zeta \in \partial \mathbb{U} \text { and }|\phi(\zeta)|=1\right\},
$$

where $\phi^{\prime}(\zeta)$ represents the usual derivative of $\phi$ at $\zeta$ and where the supremum is taken to be zero when the set over which it is taken is empty. Thus, for a nonautomorphic linear-fractional $\phi$, either $\left\|C_{\phi}\right\|_{e}=0$ (when $\phi(\partial \mathbb{U})$ lies inside $\partial \mathbb{U}$ ) or $\left\|C_{\phi}\right\|_{e}^{2}=1 /\left|\phi^{\prime}(\zeta)\right|$, where $\zeta$ is the unique point on $\partial \mathbb{U}$ for which $|\phi(\zeta)|=1$. In particular, note that if $\zeta \in \partial \mathbb{U}$ equals the Denjoy-Wolff point $\omega$ of $\phi$ and $\phi$ is non-automorphic, then (2.4) yields $\left\|C_{\phi}\right\|_{e}=\left(\phi^{\prime}(\omega)\right)^{-1 / 2}$, which in turn equals $\operatorname{rad}\left(C_{\phi}\right)$. That is, the essential norm equals the spectral radius for a linear-fractional composition operator whose symbol is non-automorphic and has its Denjoy-Wolff point on $\partial \mathbb{U}$. Thus, for example, if $\phi_{r, s}$ belongs to the Cowen-Kriete family (1.3), then $\left\|C_{\phi}\right\|_{e}=\operatorname{rad}\left(C_{\phi}\right)$. (For $-1<r<1, \phi_{r, s}$ is not an automorphism and 1 is its Denjoy-Wolff point; for $r=1, \phi_{r, s}$ is an automorphism, but (2.3) may be used to obtain the equality of essential norm and spectral radius in this case.) Observe that $\left\|C_{\phi}\right\|_{e}=1$ for a "parabolic-type" mapping, such as $\phi(z)=1 /(2-z)$, which has Denjoy-Wolff point 1 and has derivative 1 at 1 .

The following fact, proved by Hammond in [14, Propositions 2.1-2.3], constitutes the starting point for our work: if $\phi$ is an analytic self-map of $\mathbb{U}$ such that $\left\|C_{\phi}\right\|_{e}<$ $\left\|C_{\phi}\right\|$, then there is a function $g \in H^{2}$ that vanishes nowhere on $\mathbb{U}$ such that

$$
C_{\phi}^{*} C_{\phi} g=\left\|C_{\phi}\right\|^{2} g .
$$

Assuming that $\phi(z)=(a z+b) /(c z+d)$ is non-constant, one may compute $C_{\phi}^{*}$ using a formula derived by Cowen [8]:

$$
C_{\phi}^{*}=T_{\gamma} C_{\sigma} T_{\nu}^{*}
$$

where $\gamma(z)=1 /(-\bar{b} z+\bar{d}), \nu(z)=c z+d, \sigma(z)=(\bar{a} z-\bar{c}) /(-\bar{b} z+\bar{d})$, and $T_{h}$ represents the analytic Toeplitz operator with symbol $h$. Using Cowen's formula, Hammond proves that for every $f \in H^{2}$,

$$
\left(C_{\phi}^{*} C_{\phi} f\right)(z)=\psi(z) f(\tau(z))+\chi(z) f(\phi(0)), z \neq \frac{\bar{c}}{\bar{a}},
$$


where

$$
\psi(z)=\frac{(\overline{a d}-\overline{b c}) z}{(\bar{a} z-\bar{c})(-\bar{b} z+\bar{d})}, \chi(z)=\frac{\bar{c}}{-\bar{a} z+\bar{c}}, \quad \text { and } \tau(z)=\phi(\sigma(z)) .
$$

Let $f$ be an eigenvector for $C_{\phi}^{*} C_{\phi}$ with corresponding eigenvalue $\lambda$. Hammond uses (2.7), together with the easily proven fact that $f(\phi(0))=\lambda f(0)$ (see equation (2.1) of [14]), to show that the following functional equation holds:

$$
\lambda f(z)=\psi(z) f(\tau(z))+\chi(z) \lambda f(0)
$$

for all $z \in \mathbb{U}$ except for the possible singularity at $z=\bar{c} / \bar{a}$. An induction argument, based on equation (2.8), shows that, for every $j \geq 1$,

$$
\lambda^{j} f(z)=\left[\prod_{m=0}^{j-1} \psi\left(\tau^{[m]}(z)\right)\right] f\left(\tau^{[j]}(z)\right)+f(0) \sum_{k=0}^{j-1} \chi\left(\tau^{[k]}(z)\right)\left[\prod_{m=0}^{k-1} \psi\left(\tau^{[m]}(z)\right)\right] \lambda^{j-k} .
$$

Substituting $\phi(0)$ for $z$ in the preceding equation and again using the fact that $f(\phi(0))=\lambda f(0)$, one obtains

$$
\begin{aligned}
\lambda^{j+1} f(0)= & {\left[\prod_{m=0}^{j-1} \psi\left(\tau^{[m]}(\phi(0))\right)\right] f\left(\tau^{[j]}(\phi(0))\right) } \\
& +f(0) \sum_{k=0}^{j-1} \chi\left(\tau^{[k]}(\phi(0))\right)\left[\prod_{m=0}^{k-1} \psi\left(\tau^{[m]}(\phi(0))\right)\right] \lambda^{j-k},
\end{aligned}
$$

for $j \geq 1$, which is essentially Proposition 5.1 of [14].

In [14, Hammond uses equation (2.10), together with the assumption that $\tau^{[j]}(\phi(0))=0$ for some $j$, to find exact representations for the norms of certain composition operators. In the next section, we derive a " $j=\infty$ " version of (2.10) for $C_{\phi}$, given that $\left\|C_{\phi}\right\|>\left\|C_{\phi}\right\|_{e}>0$. Moreover, we show that $\left\|C_{\phi}\right\|>\left\|C_{\phi}\right\|_{e}$ when $\phi$ has the form $\phi(z)=b /(c z+d)$, which leads us to representations for the norms of all such composition operators. Note also that $\left\|C_{\phi}\right\|>\left\|C_{\phi}\right\|_{e}$ when $\phi$ is of parabolic type and not an automorphism; in this case $\left\|C_{\phi}\right\|_{e}=1$, while $\left\|C_{\phi}\right\|>1$ since $\phi(0) \neq 0$ (when $\phi$ is of parabolic type its Denjoy-Wolff point lies on $\partial \mathbb{U}$ ). Of course, $\left\|C_{\phi}\right\|$ will exceed $\left\|C_{\phi}\right\|_{e}$ when $C_{\phi}$ is compact. As we discussed above, for linear-fractional composition operators, determining when $C_{\phi}$ is compact is easy:

$$
C_{\phi} \text { is compact if and only if }\|\phi\|_{\infty}<1 \text {, }
$$

where, as usual, $\|\phi\|_{\infty}=\sup \{|\phi(z)|: z \in \mathbb{U}\}$. (Even without the hypothesis that $\phi$ be linear fractional, the condition $\|\phi\|_{\infty}<1$ trivially gives that $C_{\phi}$ is compact. The converse - for linear-fractional $\phi$ - follows immediately from the essential norm formula (2.4).)

\section{RESUlts FOR NON-COMPACT $C_{\phi}$}

Throughout this section, unless otherwise specified, $\phi$ denotes a non-constant linear-fractional self-mapping of $\mathbb{U}$ with form $\phi(z)=(a z+b) /(c z+d)$. As we pointed out in the preceding section, if $\phi$ happens to be an automorphism, then $\left\|C_{\phi}\right\|^{2}=(1+|\phi(0)|) /(1-|\phi(0)|)$. Thus we will assume throughout this section that $\phi$ is not an automorphism. Such a $\phi$ induces a non-compact composition operator if and only if there are points $\zeta$ and $\eta$ in $\partial \mathbb{U}$ such that $\phi(\zeta)=\eta$. We seek to compute $\left\|C_{\phi}\right\|$ in this situation. We claim that, without loss of generality, we may assume 
$\zeta=\eta=1$, so that $\phi$ fixes 1 . To verify the claim, note that $C_{\zeta z}$ and $C_{\bar{\eta} z}$ are unitary (given that $|\zeta|=|\eta|=1$ ) and thus $\left\|C_{\zeta z} C_{\phi} C_{\bar{\eta} z}\right\|=\left\|C_{\phi}\right\|$. Hence if $\phi(\zeta)=\eta$, then $C_{\zeta z} C_{\phi} C_{\bar{\eta} z}=C_{\bar{\eta} \phi(\zeta z)}$ is a composition operator, having the same norm as $C_{\phi}$, that is induced by a linear-fractional self-map of $\mathbb{U}$ which fixes 1 .

We will use $\tau, \chi$, and $\psi$ to denote the auxiliary mappings associated with $\phi$ by the functional equation (2.7); specifically, $\chi(z)=\bar{c} /(-\bar{a} z+\bar{c}), \tau(z)=(\phi \circ \sigma)(z)$, where $\sigma$ is the self-map of $\mathbb{U}$ in Cowen's formula (2.6) for $C_{\phi}^{*}$, and

$$
\psi(z)=\frac{(\overline{a d}-\overline{b c}) z}{(\bar{a} z-\bar{c})(-\bar{b} z+\bar{d})} .
$$

We require a number of lemmas. The first is an immediate consequence of Lemma 5.1 of [6], which shows that if $\phi$ fixes 1 , then so does $\sigma$, and $\sigma^{\prime}(1)=1 / \phi^{\prime}(1)$.

Lemma 3.1. Suppose that $\phi$ fixes 1 ; then $\tau$ fixes 1 . Moreover $\tau^{\prime}(1)=1$, so that 1 is the Denjoy-Wolff point of $\tau$.

The preceding lemma does not hold if $\phi$ is an automorphism; in this case, it is easy to check that $\sigma=\phi^{-1}$ so that $\tau$ is the identity mapping. The proof of the next lemma is a simple computation based on the formulas for $\psi$ and $\sigma$.

Lemma 3.2. For $z \in \mathbb{C} \backslash\{\bar{c} / \bar{a}, \bar{d} / \bar{b}\}$,

$$
\psi(z)=\frac{z \sigma^{\prime}(z)}{\sigma(z)} .
$$

Lemma 3.3. Suppose that $\phi$ fixes 1 . For each $z_{0} \in \mathbb{U}$, there is a constant $C$ such that for all $j \geq 1$,

$$
\frac{1}{1-\left|\tau^{[j]}\left(z_{0}\right)\right|} \leq C j
$$

Proof. Recall that we are assuming throughout this section that $\phi$ is not an automorphism. Because $\tau=\phi \circ \sigma, \tau$ is also not an automorphism. In addition, Lemma 3.1 shows that $\tau(1)=1=\tau^{\prime}(1)$, so that $\tau$ is of parabolic type. The argument establishing this lemma appears as part of the proof of Theorem 2.4 of [4]. For the convenience of the reader we sketch it. Conjugating $\tau$ with $T(z)=(1+z) /(1-z)$ produces a self-mapping of the right half-plane of the form $w \mapsto w+q$, where $\Re(q)>0$. Moving back to the disk and iterating shows that

$$
\tau^{[j]}(z)=\frac{(2-j q) z+j q}{-j q z+(2+j q)} .
$$

Let $z_{0} \in \mathbb{U}$. A computation yields $\lim _{j \rightarrow \infty} j\left(1-\tau^{[j]}\left(z_{0}\right)\right)=2 / q$. Since the convergence of $\left(\tau^{[j]}\left(z_{0}\right)\right)$ to 1 is non-tangential, there is a constant $A$ for which $\left|1-\tau^{[j]}\left(z_{0}\right)\right| \leq A\left(1-\left|\tau^{[j]}\left(z_{0}\right)\right|\right)$, and the lemma follows.

Lemma 3.4. Suppose that $\phi$ fixes the point 1 . Then for every $j \geq 0, \tau^{[j]}(\phi(0)) \neq$ $\bar{c} / \bar{a}$. In addition, $\bar{c} / \bar{a} \neq 1$.

Proof. Note that $\sigma^{-1}(0)=\bar{c} / \bar{a}$. Suppose, in order to obtain a contradiction, that $\tau^{[j]}(\phi(0))=\sigma^{-1}(0)$ for some $j \geq 0$. Then $\tau^{[j+1]}(\phi(0))=\tau\left(\sigma^{-1}(0)\right)=\phi(0)$. Thus $\tau^{[j+1]}$ fixes the point $\phi(0) \in \mathbb{U}$, but this contradicts $\tau$ 's having Denjoy-Wolff point 1 (Lemma 3.1).

The second assertion of the lemma is even easier to prove: because $\phi(1)=1$ implies $\sigma(1)=1$, we see that $\sigma^{-1}(0) \neq 1$. 
The following theorem provides a representation of the norm of $C_{\phi}$ whenever $C_{\phi}$ is not compact and $\left\|C_{\phi}\right\|>\left\|C_{\phi}\right\|_{e}$. Note that the hypothesis $\left\|C_{\phi}\right\|>\left\|C_{\phi}\right\|_{e}$ holds whenever $\phi$ has parabolic type, or, more generally, whenever $\phi^{\prime}(1) \leq 1$.

Theorem 3.5. Let $\phi$ be a linear-fractional mapping that fixes the point 1 and let $\lambda=\left\|C_{\phi}\right\|^{2}$. Suppose that $\left\|C_{\phi}\right\|>\left\|C_{\phi}\right\|_{e}$, so that $\lambda>\left\|C_{\phi}\right\|_{e}^{2}$; then

$$
\sum_{k=0}^{\infty} \chi\left(\tau^{[k]}(\phi(0))\right)\left[\prod_{m=0}^{k-1} \psi\left(\tau^{[m]}(\phi(0))\right)\right]\left(\frac{1}{\lambda}\right)^{k+1}=1 .
$$

Moreover, the largest number $\lambda$ for which (3.2) holds is the square of the norm of $C_{\phi}$.

We remark that (3.2) reduces to the polynomial equation described in 14, Theorem 5.5] if $\tau^{[j]}(\phi(0))=0$ for some $j$.

Proof of Theorem 3.5. Because $\left\|C_{\phi}\right\|>\left\|C_{\phi}\right\|_{e}$, we know from (2.5) that there is an $H^{2}$ function $g$ with $g(0) \neq 0$ such that

$$
C_{\phi}^{*} C_{\phi} g=\left\|C_{\phi}\right\|^{2} g .
$$

Thus (2.10) holds with $f=g$ and $\lambda=\left\|C_{\phi}\right\|^{2}$ : for each $j \geq 1$,

$$
\begin{aligned}
\lambda^{j+1} g(0)= & {\left[\prod_{m=0}^{j-1} \psi\left(\tau^{[m]}(\phi(0))\right)\right] g\left(\tau^{[j]}(\phi(0))\right) } \\
& +g(0) \sum_{k=0}^{j-1} \chi\left(\tau^{[k]}(\phi(0))\right)\left[\prod_{m=0}^{k-1} \psi\left(\tau^{[m]}(\phi(0))\right)\right] \lambda^{j-k} .
\end{aligned}
$$

Thus (3.2) of the theorem will follow if we can prove that

$$
\mathcal{P}_{j}:=\left(\frac{1}{\lambda}\right)^{j+1}\left[\prod_{m=0}^{j-1} \psi\left(\tau^{[m]}(\phi(0))\right)\right] g\left(\tau^{[j]}(\phi(0))\right)
$$

converges to 0 as $j \rightarrow \infty$. First observe that, by Lemma 3.4, every factor in the product

$$
\prod_{m=0}^{j-1} \psi\left(\tau^{[m]}(\phi(0))\right)
$$

is a complex number. We claim that the factors converge to $\frac{1}{\phi^{\prime}(1)}$; that is,

$$
\lim _{m \rightarrow \infty} \psi\left(\tau^{[m]}(\phi(0))\right)=\frac{1}{\phi^{\prime}(1)} .
$$

To verify the claim, note that $\tau^{[m]}(\phi(0))$ converges to 1 as $m \rightarrow \infty$ because $\tau$ has Denjoy-Wolff point 1 (Lemma 3.1). Now note that the formula for $\psi$ given by Lemma 3.2 shows that $\psi(1)=\sigma^{\prime}(1)$, which equals $1 / \phi^{\prime}(1)$ by Lemma 5.1 of [6]. Finally, Lemma 3.4 shows that $\psi$ is continuous at 1 , and our claim follows.

The essential norm $\left\|C_{\phi}\right\|_{e}$ is $1 / \sqrt{\phi^{\prime}(1)}$ by (2.4). Thus our hypothesis on $\lambda$ is that $\lambda>1 / \phi^{\prime}(1)$. By (3.4), there is a constant $\beta \in(0,1)$ and an $M>1$ such that whenever $m \geq M$,

$$
\frac{\left|\psi\left(\tau^{[m]}(\phi(0))\right)\right|}{\lambda}<\beta<1 .
$$


We have for $j>M$

$$
\begin{aligned}
\left|\mathcal{P}_{j}\right| & \leq\left[\frac{\beta^{j-M}}{\lambda} \prod_{m=0}^{M-1} \frac{\left|\psi\left(\tau^{[m]}(\phi(0))\right)\right|}{\lambda}\right]\left|g\left(\tau^{[j]}(\phi(0))\right)\right| \\
& \leq\left[\frac{\beta^{j-M}}{\lambda} \prod_{m=0}^{M-1} \frac{\left|\psi\left(\tau^{[m]}(\phi(0))\right)\right|}{\lambda}\right] \frac{\|g\|}{\left(1-\left|\tau^{[j]}(\phi(0))\right|\right)^{1 / 2}} \\
& \leq \frac{\|g\| \sqrt{C} \sqrt{j} \beta^{j-M}}{\lambda}\left[\prod_{m=0}^{M-1} \frac{\left|\psi\left(\tau^{[m]}(\phi(0))\right)\right|}{\lambda}\right] \\
& \rightarrow 0 \text { as } j \rightarrow \infty,
\end{aligned}
$$

where we have used (2.1) to bound $\left|g\left(\tau^{[j]}(\phi(0))\right)\right|$, as well as Lemma 3.3

Because $\mathcal{P}_{j} \rightarrow 0$ as $j \rightarrow 0$, we have established that (3.2) holds with $\lambda=\left\|C_{\phi}\right\|^{2}$; in other words, $\left\|C_{\phi}\right\|^{2}$ belongs to the set

$$
S:=\left\{\lambda>0: \sum_{k=0}^{\infty} \chi\left(\tau^{[k]}(\phi(0))\right)\left[\prod_{m=0}^{k-1} \psi\left(\tau^{[m]}(\phi(0))\right)\right]\left(\frac{1}{\lambda}\right)^{k+1}=1\right\} .
$$

Thus $\left\|C_{\phi}\right\|^{2} \leq \sup S$.

We claim that $S$ contains a maximum element. We have shown that the power series

$$
\sum_{k=0}^{\infty} \chi\left(\tau^{[k]}(\phi(0))\right)\left[\prod_{m=0}^{k-1} \psi\left(\tau^{[m]}(\phi(0))\right)\right] z^{k+1}
$$

has a positive radius of convergence - it converges to 1 at the positive number $1 /\left\|C_{\phi}\right\|^{2}$, for example. (In fact, it is not difficult to compute the radius of convergence exactly. Whenever $\tau^{[j]}(\phi(0))=0$ for some $j$, the series simply reduces to a polynomial. Otherwise, the radius of convergence is $\phi^{\prime}(1)=1 /\left\|C_{\phi}\right\|_{e}^{2}$; to see this, use (3.4) and the fact that $\lim _{k \rightarrow \infty} \chi\left(\tau^{[k]}(\phi(0))\right)=\chi(1)$, which is finite by Lemma 3.4.) Since the function defined by the series vanishes at $z=0$, by continuity there must be a minimum positive number $s$ at which the series converges to 1 . The maximum element in $S$ is $1 / s$. Now we show that $\left\|C_{\phi}\right\|^{2}=\max S$.

Let $\Lambda=\max S$. We have already noted that $\left\|C_{\phi}\right\|^{2} \leq \Lambda$; our goal now is to prove that $\Lambda$ belongs to the spectrum of $C_{\phi}^{*} C_{\phi}$, which will show that $\Lambda \leq\left\|C_{\phi}^{*} C_{\phi}\right\|=$ $\left\|C_{\phi}\right\|^{2}$. Suppose, to the contrary, that $\Lambda$ is not in the spectrum. In other words, the operator $C_{\phi}^{*} C_{\phi}-\Lambda$ is invertible; thus, for any $h \in H^{2}$, there is an element $f \in H^{2}$ such that

$$
C_{\phi}^{*} C_{\phi} f-\Lambda f=-h .
$$

Recalling our representation for $C_{\phi}^{*} C_{\phi}$, we see that

$$
\Lambda f(z)=\psi(z) f(\tau(z))+f(\phi(0)) \chi(z)+h(z)
$$

for all $z \in \mathbb{U}$ except possibly $z=\bar{c} / \bar{a}$. An induction argument shows that

$$
\begin{aligned}
\Lambda^{j} f(z)= & {\left[\prod_{m=0}^{j-1} \psi\left(\tau^{[m]}(z)\right)\right] f\left(\tau^{[j]}(z)\right)+\sum_{k=0}^{j-1}\left[f(\phi(0)) \chi\left(\tau^{[k]}(z)\right)\right.} \\
& \left.+h\left(\tau^{[k]}(z)\right)\right]\left[\prod_{m=0}^{k-1} \psi\left(\tau^{[m]}(z)\right)\right] \Lambda^{j-k-1}
\end{aligned}
$$


for every $j \geq 1$. We substitute $\phi(0)$ for $z$ and divide both sides of the equation by $\Lambda^{j}$; an argument identical to that from the first half of the proof shows that the terms

$$
\left(\frac{1}{\Lambda}\right)^{j}\left[\prod_{m=0}^{j-1} \psi\left(\tau^{[m]}(\phi(0))\right)\right] f\left(\tau^{[j]}(\phi(0))\right)
$$

converge to 0 as $j \rightarrow \infty$. Since $\Lambda$ belongs to $S$, we see that

$$
\begin{aligned}
f(\phi(0)) & =\sum_{k=0}^{\infty}\left[f(\phi(0)) \chi\left(\tau^{[k]}(\phi(0))\right)+h\left(\tau^{[k]}(\phi(0))\right)\right]\left[\prod_{m=0}^{k-1} \psi\left(\tau^{[m]}(\phi(0))\right)\right]\left(\frac{1}{\Lambda}\right)^{k+1} \\
& =f(\phi(0))+\sum_{k=0}^{\infty} h\left(\tau^{[k]}(\phi(0))\right)\left[\prod_{m=0}^{k-1} \psi\left(\tau^{[m]}(\phi(0))\right)\right]\left(\frac{1}{\Lambda}\right)^{k+1} .
\end{aligned}
$$

In other words,

$$
\sum_{k=0}^{\infty} h\left(\tau^{[k]}(\phi(0))\right)\left[\prod_{m=0}^{k-1} \psi\left(\tau^{[m]}(\phi(0))\right)\right]\left(\frac{1}{\Lambda}\right)^{k+1}=0
$$

for all $h \in H^{2}$

We hope to use this fact to obtain a contradiction. Indeed, if $|c|>|a|$, our task is quite simple. In this case, the function $\chi$ belongs to $H^{2}$; taking $\chi$ for $h$ in (3.5), we obtain a contradiction to the membership of $\Lambda$ in $S$. We must be a bit more careful when $|c| \leq|a|$. To begin with, let $K$ be a simply connected, compact subset of $\mathbb{C}$ which does not contain $\bar{c} / \bar{a}$ but which does contain all of the points $\tau^{[m]}(\phi(0))$, $m=0,1,2, \ldots$. Since $\chi$ is analytic in the open set $\mathbb{C} \backslash\{\bar{c} / \bar{a}\}$, it follows from [18. Theorem 13.7] that there is a sequence of polynomials $\left(p_{n}\right)$ which converges to $\chi$ uniformly on $K$. Since every polynomial belongs to $H^{2}$, (3.5) holds for each $p_{n}$. Now we make the following observation:

$$
\begin{aligned}
& \mid \sum_{k=0}^{\infty} \chi\left(\tau^{[k]}(\phi(0))\right)\left[\prod_{m=0}^{k-1} \psi\left(\tau^{[m]}(\phi(0))\right)\right]\left(\frac{1}{\Lambda}\right)^{k+1} \\
& \quad-\sum_{k=0}^{\infty} p_{n}\left(\tau^{[k]}(\phi(0))\right)\left[\prod_{m=0}^{k-1} \psi\left(\tau^{[m]}(\phi(0))\right)\right]\left(\frac{1}{\Lambda}\right)^{k+1} \mid \\
& \quad \leq \sum_{k=0}^{\infty}\left|\chi\left(\tau^{[k]}(\phi(0))\right)-p_{n}\left(\tau^{[k]}(\phi(0))\right)\right|\left[\prod_{m=0}^{k-1}\left|\psi\left(\tau^{[m]}(\phi(0))\right)\right|\right]\left(\frac{1}{\Lambda}\right)^{k+1} .
\end{aligned}
$$

Recall that the sequence $\left(\psi\left(\tau^{[m]}(\phi(0))\right)\right)$ converges to $\psi(1)=1 / \phi^{\prime}(1)=\left\|C_{\phi}\right\|_{e}^{2}$ as $m \rightarrow \infty$. Since $\Lambda>\left\|C_{\phi}\right\|_{e}^{2}$, it follows that there is a constant $A>0$ and a constant $\gamma$ with $0<\gamma<1$ such that

$$
\prod_{m=0}^{k-1} \frac{\left|\psi\left(\tau^{[m]}(\phi(0))\right)\right|}{\Lambda}<A \gamma^{k}
$$

for every $k \geq 0$. Therefore expression (3.6) is bounded by

$$
\sum_{k=0}^{\infty}\left|\chi\left(\tau^{[k]}(\phi(0))\right)-p_{n}\left(\tau^{[k]}(\phi(0))\right)\right|\left(\frac{A}{\Lambda}\right) \gamma^{k} .
$$


Since the sequence $\left(p_{n}\right)$ converges to $\chi$ uniformly on $K$, we conclude that

$$
\sum_{k=0}^{\infty} \chi\left(\tau^{[k]}(\phi(0))\right)\left[\prod_{m=0}^{k-1} \psi\left(\tau^{[m]}(\phi(0))\right)\right]\left(\frac{1}{\Lambda}\right)^{k+1}=0,
$$

which is a contradiction.

The next corollary, which follows from the proof of the preceding theorem, provides a means for showing that the norm of $C_{\phi}$ exceeds the essential norm.

Corollary 3.6. Let $\phi$ be a (non-automorphic) linear-fractional mapping that fixes the point 1 . If there is a number $\Lambda$ such that $\Lambda>1 / \phi^{\prime}(1)$ and

$$
1=\sum_{k=0}^{\infty} \chi\left(\tau^{[k]}(\phi(0))\right)\left[\prod_{m=0}^{k-1} \psi\left(\tau^{[m]}(\phi(0))\right)\right]\left(\frac{1}{\Lambda}\right)^{k+1},
$$

then $\Lambda$ belongs to the spectrum of $C_{\phi}^{*} C_{\phi}$; hence, in particular, $\left\|C_{\phi}\right\|^{2}=\left\|C_{\phi}^{*} C_{\phi}\right\| \geq$ $\Lambda>1 / \phi^{\prime}(1)=\left\|C_{\phi}\right\|_{e}^{2}$.

As an application of the preceding corollary, we obtain the following information for composition operators induced by members of the Cowen-Kriete family.

Theorem 3.7. Suppose that

$$
\phi_{r, s}(z)=\frac{(r+s) z+1-s}{r(1-s) z+1+s r},
$$

where $0<s<1$ and $-1<r<0$. Then $\left\|C_{\phi_{r, s}}\right\|>\left\|C_{\phi_{r, s}}\right\|_{e}=\operatorname{rad}\left(C_{\phi_{r, s}}\right)$, so that $C_{\phi_{r, s}}^{*}$ is not hyponormal.

Proof. As discussed in Section 2, $\left\|C_{\phi_{r, s}}\right\|_{e}=\operatorname{rad}\left(C_{\phi_{r, s}}\right)$. Thus, to prove the theorem, we need only show that the norm exceeds the essential norm. By Corollary 3.6 we may accomplish this by showing that there exists a number $\Lambda>1 / \phi_{r, s}^{\prime}(1)=1 / s$ for which (3.2) holds:

$$
1=\sum_{k=0}^{\infty} \chi\left(\tau^{[k]}\left(\phi_{r, s}(0)\right)\right)\left[\prod_{m=0}^{k-1} \psi\left(\tau^{[m]}\left(\phi_{r, s}(0)\right)\right)\right]\left(\frac{1}{\Lambda}\right)^{k+1},
$$

where $\chi, \tau$, and $\psi$ are the usual auxiliary functions for $\phi_{r, s}$. Make the substitution $x=1 / \Lambda$ in (3.2) and consider

$$
f(x)=\sum_{k=0}^{\infty} a_{k} x^{k+1}
$$

where for $k \geq 0$,

$$
a_{k}=\chi\left(\tau^{[k]}\left(\phi_{r, s}(0)\right)\right)\left[\prod_{m=0}^{k-1} \psi\left(\tau^{[m]}\left(\phi_{r, s}(0)\right)\right)\right] .
$$

We claim that $a_{k}>0$ for each $k \geq 0$. To verify the claim, we will show that $\chi(x)$ and $\psi(x)$ are positive for $0<x \leq 1$. The positivity of the $a_{k}$ 's will then follow, since every element of the sequence $\left(\tau^{[m]}\left(\phi_{r, s}(0)\right)\right)$ is easily seen to be positive: the sequence "starts out positive", $\tau^{[0]}\left(\phi_{r, s}(0)\right)=\phi_{r, s}(0)=(1-s) /(1+s r)>0$, and further iterations push the sequence along the positive real axis toward 1 , the Denjoy-Wolff point of $\tau$. 
We know that

$$
\chi(z)=\frac{r(1-s)}{r(1-s)-(r+s) z} .
$$

Observe that $\chi(x)$ will be real (or infinite) if $x$ is real; also observe that we may show that $\chi$ is positive for $x \in(0,1]$ by showing its denominator $r(1-s)-(r+s) x$ is negative for such $x$ (the numerator of $\chi$ is negative because we are assuming $r<0$ and $0<s<1)$. Let $d(x)=r(1-s)-(r+s) x$ be the "denominator function" for $\chi$; then $d(0)=r(1-s)<0$ and $d(1)=-s(1+r)<0$. Thus, if $d$ has no zero in $(0,1)$, our proof that $\chi$ is positive on $(0,1]$ is complete. The only zero of $d$ is $r(1-s) /(r+s)$. Suppose, in order to obtain a contradiction, that

$$
0<r(1-s) /(r+s)<1 .
$$

Because $r<0$, the left inequality in (3.7) yields $r+s<0$. Now multiply both sides of the right inequality of (3.7) by $r+s$ to see that $r(1-s)>r+s$, or $0>s(1+r)$, a contradiction.

We know that

$$
\psi(z)=\frac{s(r+1)^{2} z}{((-1+s) z+1+s r)((r+s) z-r+s r)} .
$$

The numerator of $\psi(x)$ is clearly positive if $0<x<1$; we must show that the same is true of the denominator. The denominator of $\psi(x)$ is a quadratic polynomial with zeros $b_{1}=(1+s r) /(1-s)$ and $b_{2}=r(1-s) /(r+s)$. Note that $b_{1}$ exceeds 1 , while $b_{2}$ cannot lie in the interval $(0,1)$ by the work of the preceding paragraph. The denominator is positive at $0,(1+s r)(-r+s r)>0$, and positive at $1,(s+s r)^{2}>0$. Our proof that $\psi$ is positive on $(0,1]$ is complete.

As we pointed out in the proof of Theorem [3.5, the radius of convergence of the series defining $f$ is $\phi_{r, s}{ }^{\prime}(1)$, which here equals $s$. In fact, $\lim _{k \rightarrow \infty}\left(a_{k}\right)^{1 / k}=1 / s$. Note that $f(0)=0$ and that, because $a_{k}>0$ for $k \geq 0, f(x)>0$ for $0<x<s$. We will show that $f(x) \rightarrow \infty$ as $x \rightarrow s^{-}$. It will follow that there is a real number $\alpha$ with $0<\alpha<s$ such that $f(\alpha)=1$. If we set $\Lambda=1 / \alpha$, we have the desired $\Lambda$ that satisfies (3.2) and exceeds $1 / \phi_{r, s}{ }^{\prime}(1)=1 / s$.

We claim that there is a positive constant $c$ such that for every $k \geq 0$,

$$
(k+1) a_{k} s^{k+1} \geq c .
$$

Thus $f(x) \geq c \sum_{k=0}^{\infty} \frac{1}{k+1}\left(\frac{x}{s}\right)^{k+1}$ for $0<x<s$, from which we may conclude $f(x) \rightarrow \infty$ as $x \rightarrow s^{-}$. Consider the definition of $a_{k}$ and note that $\left(\chi\left(\tau^{[k]}\left(\phi_{r, s}(0)\right)\right)\right)$ is a sequence of positive numbers converging to a positive number. Thus, to establish the inequality (3.8), it suffices to prove

$$
(k+1) \prod_{m=0}^{k-1}\left[\psi\left(\tau^{[m]}\left(\phi_{r, s}(0)\right)\right) s\right] \geq c,
$$

for some constant $c$ and all $k \geq 0$. A little calculus shows that each factor in the product (3.9) is less than 1 (i.e., $\psi$ is increasing on $(0,1)$ and $\psi(1)=1 / s$ while $0<\tau^{[m]}\left(\phi_{r, s}(0)\right)<1$ for every $\left.m\right)$. Now take the logarithm of the quantity on the left of (3.9):

$$
\log (k+1)+\sum_{m=0}^{k-1} \log \left[\psi\left(\tau^{[m]}\left(\phi_{r, s}(0)\right)\right) s\right]
$$


If we can show that the preceding real-valued function of $k$ is bounded below, then (3.9) will follow and our proof will be complete.

The right half-plane version of $\tau$, obtained by conjugating $\tau$ with $T(z)=$ $(1+z) /(1-z)$, is given by

$$
w \mapsto w+2 \frac{-r+s r+1-s}{s(1+r)} .
$$

Let us set $b=2 \frac{-r+s r+1-s}{s(1+r)}$. Calculating, just as in the proof of Lemma 3.3, we have

$$
1-\tau^{[m]}\left(\phi_{r, s}(0)\right)=\frac{2\left(1-\phi_{r, s}(0)\right)}{m b\left(1-\phi_{r, s}(0)\right)+2} .
$$

Observe that $\lim _{m \rightarrow \infty}(m+1)\left(1-\tau^{[m]}\left(\phi_{r, s}(0)\right)\right)=2 / b$, so that there is a constant $C>0$ such that

$$
\left|1-\tau^{[m]}\left(\phi_{r, s}(0)\right)\right| \leq C /(m+1) \text { for all } m \geq 0 .
$$

Another quantity we must compute is $\psi^{\prime}(1)$ :

$$
\psi^{\prime}(1)=\frac{-r+s r+1-s}{s^{2}(1+r)}=\frac{b}{2 s} .
$$

Finally, using the analyticity of $\psi$ near 1 , we conclude that

$$
s \psi(z)=1+s \psi^{\prime}(1)(z-1)+\Gamma(z),
$$

where $\Gamma$ is meromorphic (having two simple poles corresponding to those of $\psi$ ) and $\Gamma(z)=O\left(|z-1|^{2}\right)$ as $z \rightarrow 1$. We use the elementary inequality $\log (x) \geq(1-1 / x)$, $0<x<1$, to obtain the initial inequality in the next computation and use our series representation for $s \psi$ to obtain the second equality.

$$
\begin{aligned}
\sum_{m=0}^{k-1} \log \left[\psi\left(\tau^{[m]}\left(\phi_{r, s}(0)\right)\right) s\right] \geq & \sum_{m=0}^{k-1}\left(1-\frac{1}{\psi\left(\tau^{[m]}\left(\phi_{r, s}(0)\right)\right) s}\right) \\
= & \sum_{m=0}^{k-1} \frac{s \psi\left(\tau^{[m]}\left(\phi_{r, s}(0)\right)\right)-1}{s \psi\left(\tau^{[m]}\left(\phi_{r, s}(0)\right)\right)} \\
= & \sum_{m=0}^{k-1} \frac{s \psi^{\prime}(1)\left(\tau^{[m]}\left(\phi_{r, s}(0)\right)-1\right)}{s \psi\left(\tau^{[m]}\left(\phi_{r, s}(0)\right)\right)} \\
& +\sum_{m=0}^{k-1} \frac{\Gamma\left(\tau^{[m]}\left(\phi_{r, s}(0)\right)\right)}{s \psi\left(\tau^{[m]}\left(\phi_{r, s}(0)\right)\right)} \\
= & S_{1}(k)+S_{2}(k) .
\end{aligned}
$$

The second sum $S_{2}(k)$ can do no harm: it is a bounded function of $k$. The denominators of the summands in this second sum converge to 1 and $\left|\Gamma\left(\tau^{[m]}\left(\phi_{r, s}(0)\right)\right)\right| \leq$ $C\left|\tau^{[m]}\left(\phi_{r, s}(0)\right)-1\right|^{2} \leq C_{1} /(m+1)^{2}$ for some constants $C$ and $C_{1}$ (by definition of 
$\Gamma$ and by (3.12)). We continue with $S_{1}(k)$.

$$
\begin{aligned}
S_{1}(k) & =\sum_{m=0}^{k-1} \frac{s \psi^{\prime}(1)\left(\tau^{[m]}\left(\phi_{r, s}(0)\right)-1\right)}{s \psi\left(\tau^{[m]}\left(\phi_{r, s}(0)\right)\right)} \\
& =\sum_{m=0}^{k-1}\left(\frac{s \psi^{\prime}(1)(m+1)\left(\tau^{[m]}\left(\phi_{r, s}(0)\right)-1\right)}{s \psi\left(\tau^{[m]}\left(\phi_{r, s}(0)\right)\right)}\right) \frac{1}{m+1} \\
& =\sum_{m=0}^{k-1}\left(\frac{s \psi^{\prime}(1)(m+1)\left(\tau^{[m]}\left(\phi_{r, s}(0)\right)-1\right)}{s \psi\left(\tau^{[m]}\left(\phi_{r, s}(0)\right)\right)}+1\right) \frac{1}{m+1}-\sum_{m=0}^{k-1} \frac{1}{m+1} \\
& =S_{3}(k)-S_{4}(k) .
\end{aligned}
$$

We will now show that $S_{3}(k)$ can also do no harm: it too is a bounded function of $k$. We focus on the first factor of the $m$-th summand of $S_{3}(k)$.

$$
\begin{aligned}
& \frac{s \psi^{\prime}(1)(m+1)\left(\tau^{[m]}\left(\phi_{r, s}(0)\right)-1\right)}{s \psi\left(\tau^{[m]}\left(\phi_{r, s}(0)\right)\right)}+1 \\
& \quad=\frac{\left[s \psi^{\prime}(1)(m+1)\left(\tau^{[m]}\left(\phi_{r, s}(0)\right)-1\right)+1\right]+\left[s \psi\left(\tau^{[m]}\left(\phi_{r, s}(0)\right)\right)-1\right]}{s \psi\left(\tau^{[m]}\left(\phi_{r, s}(0)\right)\right)} .
\end{aligned}
$$

Because the denominator of the preceding quantity converges to 1 as $m \rightarrow \infty$, we concern ourselves only with bounding the bracketed quantities in the numerator.

We have

$$
\begin{aligned}
\left|s \psi^{\prime}(1)(m+1)\left(\tau^{[m]}\left(\phi_{r, s}(0)\right)-1\right)+1\right| \\
=\left|-s \psi^{\prime}(1)(m+1) \frac{2\left(1-\phi_{r, s}(0)\right)}{m b\left(1-\phi_{r, s}(0)\right)+2}+1\right| \\
=\left|-(b / 2)(m+1) \frac{2\left(1-\phi_{r, s}(0)\right)}{m b\left(1-\phi_{r, s}(0)\right)+2}+1\right| \\
=\left|\frac{-b+b \phi_{r, s}(0)+2}{2+b m\left(1-\phi_{r, s}(0)\right)}\right| \\
\leq \frac{2+b\left(1-\phi_{r, s}(0)\right)}{b m\left(1-\phi_{r, s}(0)\right)}
\end{aligned}
$$

where we used (3.11) to obtain the first equality. To bound the other bracketed quantity, we use our series representation for $s \psi$ :

$$
\begin{aligned}
\left|s \psi\left(\tau^{[m]}\left(\phi_{r, s}(0)\right)\right)-1\right| & =\left|s \psi^{\prime}(1)\left(\tau^{[m]}\left(\phi_{r, s}(0)\right)-1\right)+\Gamma\left(\tau^{[m]}\left(\phi_{r, s}(0)\right)\right)\right| \\
& \leq C\left(\left|\tau^{[m]}\left(\phi_{r, s}(0)\right)-1\right|+\left|\tau^{[m]}\left(\phi_{r, s}(0)\right)-1\right|^{2}\right) \\
& \leq C_{1} /(m+1)
\end{aligned}
$$

for some positive constants $C$ and $C_{1}$, by (3.12) and the definition of $\Gamma$. Our work with the first factor in each summand of $S_{3}(k)$ shows that, for each $m \geq 0$, this factor is bounded above by a constant times $1 /(m+1)$; thus $\left|S_{3}(k)\right|$ itself is less than or equal to that constant times $\sum_{m=0}^{k-1}(m+1)^{-2}<\sum_{m=0}^{\infty}(m+1)^{-2}<\infty$. 
Summarizing, we have shown that the logarithm of the left-hand side of (3.9) (which we wish to show is bounded below independently of $k$ ) is bounded below by

$$
\log (k+1)+S_{2}(k)+S_{3}(k)-\sum_{m=0}^{k-1} \frac{1}{m+1},
$$

where $S_{2}(k)$ and $S_{3}(k)$ are bounded functions of $k$. Because $\sum_{m=0}^{k-1} \frac{1}{m+1} \leq 1+\log (k)$ for $k \geq 1$, the quantity (3.13) is bounded below independently of $k$, as desired.

As we discussed in the Introduction, the preceding result, which shows $\left\|C_{\phi_{r} s}\right\|>$ $\left\|C_{\phi_{r, s}}\right\|_{e}$ when $-1<r<0$ and $0<s<1$, is sharp in the sense that $\left\|C_{\phi_{r, s}}\right\|=$ $\left\|C_{\phi_{r, s}}\right\|_{e}$ for $0 \leq r \leq 1$. In the Introduction, we deduced the equality of norm and essential norm for the range $0 \leq r \leq 1$ from the subnormality of $C_{\phi_{r, s}}^{*}$. We remark that it is possible to use Theorem 3.5 to prove $\left\|C_{\phi_{r, s}}\right\|=\left\|C_{\phi_{r, s}}\right\|_{e}$ when $0 \leq r \leq 1$ : in this situation, one can show that the coefficients of the power series in $(1 / \lambda)$ on the left of (3.2) are all negative, so that (3.2) has no positive solutions.

We now use Theorems 3.5 and 3.7 to obtain an explicit representation for the norm of $C_{\phi}$, given that $\phi(z)=b /(c z+d)$ is a self-map of $\mathbb{U}$ inducing a noncompact composition operator. Without loss of generality, assume, as above, that $\phi(1)=1$. Then, letting $b_{1}=-b / c$ and $d_{1}=-d / c$, we have that $\phi(z)=b_{1} /\left(d_{1}-z\right)$; our hypothesis that $\phi(1)=1$ tells us that $b_{1}=d_{1}-1$. Thus $\phi$ has the form $\phi(z)=\left(d_{1}-1\right) /\left(d_{1}-z\right)$. Because $\phi$ is a self-map of $\mathbb{U}$ that fixes $1, \phi^{\prime}(1)$ is positive, from which it follows that $d_{1}$ must be positive. In fact, we must have $d_{1}>1$ in order for $\phi$ to be a non-constant analytic self-map of $\mathbb{U}$. Summarizing, we see that if we can compute the norm of $C_{\phi_{\alpha}}$ where

$$
\phi_{\alpha}(z)=\frac{\alpha-1}{\alpha-z}, \alpha>1
$$

then we can compute the norm of any non-compact linear-fractional composition operator induced by a mapping of the form $\phi(z)=b /(c z+d)$.

We claim that $\left\|C_{\phi_{\alpha}}\right\|>\left\|C_{\phi_{\alpha}}\right\|_{e}$ for every $\alpha>1$. Observe that $\phi_{\alpha}^{\prime}(1) \geq 1$ when $1<\alpha \leq 2$, so that, by (2.4), $\left\|C_{\phi_{\alpha}}\right\|_{e} \leq 1$ for this range of $\alpha$ values. On the other hand, the general estimate (2.2) shows that $\left\|C_{\phi_{\alpha}}\right\|$ always exceeds 1 . Thus our claim holds when $\alpha \leq 2$. For $\alpha>2$, the mapping $\phi_{\alpha}$ belongs to the Cowen-Kriete family: $\phi_{\alpha}=\phi_{r, s}$, where $r=-1 /(\alpha-1)$ and $s=1 /(\alpha-1)$; thus Theorem 3.7 yields the claim for $\alpha>2$.

Because $\left\|C_{\phi_{\alpha}}\right\|>\left\|C_{\phi_{\alpha}}\right\|_{e}$, we may calculate the norm of $C_{\phi_{\alpha}}$ using Theorem 3.5, that is, $\left\|C_{\phi_{\alpha}}\right\|^{2}$ is the maximum $\lambda$ satisfying (3.2). We need a formula for the coefficients in (3.2).

Lemma 3.8. Suppose that $\psi$ and $\tau$ are the auxiliary mappings of 2.7 corresponding to $\phi_{\alpha}$. Then for each $k \geq 0$,

$$
\prod_{m=0}^{k-1} \psi\left(\tau^{[m]}(\phi(0))\right)=\frac{(\alpha-1)^{k+1}}{(k+1) \alpha-1} .
$$

Proof. The $k=0$ case corresponds to the convention that an empty product should be taken to be 1 . The argument for $k \geq 1$ is inductive, depending upon the formulas for $\tau$ and $\psi$ corresponding to $\phi_{\alpha}$, for which $a=0, b=\alpha-1, c=-1$, and $d=\alpha$ :

$$
\tau(z)=\frac{(\alpha-1) z-\alpha}{z \alpha-\alpha-1} \text { and } \psi(z)=\frac{(\alpha-1) z}{-(\alpha-1) z+\alpha} .
$$


As in the proof of Lemma 3.3, $\tau^{[m]}(z)=\frac{(m \alpha-1) z-m \alpha}{z m \alpha-m \alpha-1}$. Thus

$$
\psi\left(\tau^{[m]}(z)\right)=\frac{(\alpha-1)[(m \alpha-1) z-m \alpha]}{((m+1) \alpha-1) z-(m+1) \alpha},
$$

which quickly yields

$$
\prod_{m=0}^{k-1} \psi\left(\tau^{[m]}(z)\right)=\frac{(\alpha-1)^{k} z}{-(k \alpha-1) z+k \alpha} .
$$

Substituting $z=\phi(0)=(\alpha-1) / \alpha$ into the preceding formula, we obtain (3.15), as desired.

Theorem 3.9. Suppose that $\phi_{\alpha}(z)=(\alpha-1) /(\alpha-z)$ for some $\alpha>1$. Then $\left\|C_{\phi_{\alpha}}\right\|>\left\|C_{\phi_{\alpha}}\right\|_{e}$, and $\left\|C_{\phi_{\alpha}}\right\|^{2}$ is the reciprocal of the unique solution of

$$
\sum_{k=0}^{\infty} \frac{((\alpha-1) x)^{k+1}}{(k+1) \alpha-1}=1, \quad 0<x<\frac{1}{\alpha-1} .
$$

Proof. Fix $\alpha>1$. We have already proved that $\left\|C_{\phi_{\alpha}}\right\|>\left\|C_{\phi_{\alpha}}\right\|_{e}$. Thus, by Theorem 3.5, $\left\|C_{\phi_{\alpha}}\right\|^{2}$ is the largest $\lambda$ for which

$$
\sum_{k=0}^{\infty} \chi\left(\tau^{[k]}(\phi(0))\right)\left[\prod_{m=0}^{k-1} \psi\left(\tau^{[m]}(\phi(0))\right)\right]\left(\frac{1}{\lambda}\right)^{k+1}=1,
$$

where $\chi, \psi$, and $\tau$ are the usual auxiliary functions associated with $\phi_{\alpha}$. Here $\chi \equiv 1$, so that this equation simplifies to

$$
\sum_{k=0}^{\infty} \frac{((\alpha-1) / \lambda)^{k+1}}{(k+1) \alpha-1}=1
$$

by Lemma 3.8. Thus (3.16) has a solution, namely $1 /\left\|C_{\phi}\right\|^{2}$; its uniqueness follows from the positivity of the coefficients in the power series on the left of (3.16).

Corollary 3.10. Suppose that $\phi(z)=b /(c z+d)$ is a self-map of $\mathbb{U}$; then $\left\|C_{\phi}\right\|>$ $\left\|C_{\phi}\right\|_{e}$.

Proof. If $C_{\phi}$ is compact, the result is trivial. If $C_{\phi}$ is not compact, then we may assume that $\phi=\phi_{\alpha}$ for some $\alpha>1$ and the result follows immediately from Theorem 3.9,

The next corollary confirms a conjecture of Retsek [17] p. 50].

Corollary 3.11. Suppose that $\phi(z)=b /(c z+d)$ is a self-map of $\mathbb{U}$; then $\left\|C_{\phi}\right\|$ is not given by the action of either $C_{\phi}$ or $C_{\phi}^{*}$ on the reproducing kernel functions of $H^{2}$.

Proof. The action of either $C_{\phi}$ or $C_{\phi}^{*}$ on the reproducing kernels of $H^{2}$ determines $\left\|C_{\phi}\right\|$ if and only if $\left\|C_{\phi}\right\|=\left\|C_{\phi}\right\|_{e}$ ([14, Theorem 4.4]; see also [5. Proposition 3.4]); thus this result follows immediately from Corollary 3.10 above.

We conclude this section by exhibiting explicit representations for the norms of $C_{\phi_{\alpha}}$ when $\alpha=2$ and $\alpha=3$. Suppose $\alpha=2$, so that $\phi_{2}(z)=1 /(2-z)$. By Theorem 
3.9, $\left\|C_{\phi_{2}}\right\|^{2}$ is the reciprocal of the unique positive solution to $\sum_{k=0}^{\infty} \frac{x^{k+1}}{2 k+1}=1$. Summing the series and making the substitution $x=1 /\left\|C_{\phi_{2}}\right\|^{2}$, we obtain

$$
\frac{1}{2\left\|C_{\phi_{2}}\right\|} \log \left(\frac{\left\|C_{\phi_{2}}\right\|+1}{\left\|C_{\phi_{2}}\right\|-1}\right)=1
$$

Solving the preceding equation numerically, one finds $\left\|C_{\phi_{2}}\right\| \approx 1.19968$. More interesting is the following.

Proposition 3.12. $\left\|C_{\phi_{2}}\right\|$ is a transcendental number.

Proof. Suppose that $y:=\left\|C_{\phi_{2}}\right\|$ is algebraic; then $\frac{1}{2 y} \log \left(\frac{y+1}{y-1}\right)$ has the form $\gamma \log (\beta)$, where both $\gamma$ and $\beta$ are algebraic. Any non-zero number of this form is transcendental by Baker's Theorem [3, Theorem 2.2]. However, we know that $\frac{1}{2 y} \log \left(\frac{y+1}{y-1}\right)=1$ and 1 is algebraic. Thus $y$, the norm of $C_{\phi_{2}}$, must be transcendental.

We now calculate the norm of the composition operator induced by $\phi_{3}(z)=$ $2 /(3-z)$. By Theorem 3.9, the norm is the reciprocal of the the unique positive number $x$ for which $\sum_{k=0}^{\infty} \frac{(2 x)^{k+1}}{3 k+2}=1$. Summing the series, we have

$$
(2 x)^{1 / 3} F\left((2 x)^{1 / 3}\right)+\frac{\sqrt{3} \pi}{18}(2 x)^{1 / 3}=1,
$$

where $F(t)=-(1 / 3) \log (1-t)+(1 / 6) \log \left(t^{2}+t+1\right)-(1 / \sqrt{3}) \arctan ((2 t+1) / \sqrt{3})$. Solving numerically, we obtain $\left\|C_{\phi_{3}}\right\|=\sqrt{1 / x} \approx \sqrt{2.2021}$. In [1], a lower bound of $\sqrt{2.194}$ was obtained for this norm; our representation for the norm answers [1]. Question 4.5].

\section{Results FOR COMPACT $C_{\phi}$}

Despite the fact that $\left\|C_{\phi}\right\|>\left\|C_{\phi}\right\|_{e}$ whenever $C_{\phi}$ is compact, the methods of the preceding section do not always apply in this situation. The following example illustrates why this is the case. Let

$$
\phi(z)=\frac{4 z+4}{z+12}
$$

Observe that $\|\phi\|_{\infty}=8 / 13<1$, so that $C_{\phi}$ is compact. The general norm estimate (2.2) shows that $\left\|C_{\phi}\right\|^{2} \leq 2$. Now let $\tau$ and $\psi$ be the auxiliary mappings in (2.7) associated with $\phi$, so that

$$
\psi(z)=\frac{11 z}{(4 z-1)(-z+3)}
$$

and $\tau=\phi \circ \sigma$, where $\sigma(z)=(4 z-1) /(-4 z+12)$. Thus $\tau(z)=4 /(13-4 z)$. Of course, $\|\tau\|_{\infty}<1$ so that $\tau$ 's Denjoy Wolff point $\omega$ lies in $\mathbb{U}$; a calculation shows $\omega=13 / 8-(1 / 8) \sqrt{105}$. The sequence $\tau^{[j]}(\phi(0))$ converges to $\omega$ as $j \rightarrow \infty$. Thus, as $j \rightarrow \infty$,

$$
\psi\left(\tau^{[j]}(\phi(0))\right) \rightarrow \frac{22(13-\sqrt{105})}{(11-\sqrt{105})(11+\sqrt{105})}>\frac{22(13-11)}{(11-10)(11+11)}=2 .
$$


Thus, because $\lambda=\left\|C_{\phi}\right\|^{2} \leq 2$, we see that $\lim _{j \rightarrow \infty} \psi\left(\tau^{[j]}(\phi(0))\right) / \lambda$ cannot be less than 1. On the other hand, for any self-map of $\mathbb{U}$ satisfying the hypotheses of Theorem 3.5 the corresponding limit is less than 1 , which yields a key part of the proof of Theorem 3.5: $\lim _{j \rightarrow \infty} \mathcal{P}_{j}=0$. Thus we are unable to represent $\left\|C_{\phi}\right\|$ in terms of an equation like (3.2) of Theorem 3.5. In fact, if $\psi, \tau$, and $\chi$ are the usual auxiliary functions for $\phi(z)=(4 z+4) /(z+12)$, then $\lambda:=\left\|C_{\phi}\right\|^{2}$ cannot satisfy $(3.2)$; one can show that the coefficients of the power series in $(1 / \lambda)$ on the left of (3.2) are all negative in this case.

Nevertheless, the methods of the preceding section will allow us to calculate the norms of certain compact composition operators, for instance those induced by mappings of the form $\phi(z)=b /(c z+d)$, where $\|\phi\|_{\infty}<1$. We will, in fact, obtain norm information for a larger collection of composition operators, which includes those whose symbols are "scaled-down" members of the sub-family of Cowen-Kriete mappings appearing in Theorem 3.7 .

Let $\phi$ be a linear-fractional self-map of $\mathbb{U}$ with $\|\phi\|_{\infty}=1$. For every $t$ in $(0,1]$, we define the map $\phi_{t}(z)=t \phi(z)$; except for $t=1$, each $\phi_{t}$ induces a compact composition operator. Let $\sigma_{t}, \tau_{t}, \psi_{t}$, and $\chi_{t}$ denote the usual auxiliary functions for $\phi_{t}$; let $\omega_{t}$ denote the Denjoy-Wolff point of $\tau_{t}$. (If we omit the label $t$, we mean that $t=1$.)

In light of Theorem [3.7, we can prove the following lemma.

Lemma 4.1. Suppose that $\phi=\phi_{r, s}$ for some $s \in(0,1)$ and $r \in(-1,0)$, where $\phi_{r, s}$ is given by (1.3). Then, for every $t$ in $(0,1]$, the quantity $\psi_{t}\left(\omega_{t}\right)$ is a positive real number and

$$
\psi_{t}\left(\omega_{t}\right)<\left\|C_{\phi_{t}}\right\|^{2}
$$

Proof. First of all, consider the points $\omega_{t}$. Since each map $\tau_{t}$ takes a real number to a real number, every point $\omega_{t}$ must be real; otherwise the attractive property of $\omega_{t}$ would force $\tau_{t}$ to take some real number to a non-real number. Each $\omega_{t}$ is the unique root in the closed unit disk of the quadratic equation

$$
t(1-s)(r-1) z^{2}+\left(1-r+2 s r+t^{2}(1-r-2 s)\right) z+t(1-s)(r-1)=0
$$

hence the points $\omega_{t}$ vary continuously with $t$. Moreover, since $\omega_{1}=1$, it follows that each $\omega_{t}$ is positive; otherwise $\omega_{t_{0}}$ would equal 0 for some $t_{0}$, meaning that $t_{0}(1-s)(r-1)=0$, which is not the case.

Now consider the functions $\psi_{t}$. Observe that $\psi_{t}(z)=\psi(t z)$. We showed in the proof of Theorem 3.7 that the function $\psi(x)$ is positive and continuous on the interval $(0,1]$. Hence $\psi_{t}\left(\omega_{t}\right)=\psi\left(t \omega_{t}\right)>0$ for all $t$ in $(0,1]$, and the values $\psi_{t}\left(\omega_{t}\right)$ vary continuously with $t$. Thus we see that

$$
\lim _{t \rightarrow 1^{-}} \psi_{t}\left(\omega_{t}\right)=\psi(1)=\left\|C_{\phi}\right\|_{e}^{2}<\left\|C_{\phi}\right\|^{2} \leq \liminf _{t \rightarrow 1^{-}}\left\|C_{\phi_{t}}\right\|^{2}
$$

where the first inequality follows from Theorem 3.7 and the second can be deduced from Fatou's lemma. In other words, there is some $\epsilon>0$ such that $\psi_{t}\left(\omega_{t}\right)<\left\|C_{\phi_{t}}\right\|^{2}$ for all $t$ in $(1-\epsilon, 1]$.

Whenever $0<t<1$, the map $\phi_{t}$ has the property that $\left\|\phi_{t}\right\|_{\infty}<1$. Therefore Theorem 2 of [15] dictates that the values $\left\|C_{\phi_{t}}\right\|$ vary continuously with $t \in(0,1)$. 
Consequently

$$
M(t):=\psi_{t}\left(\omega_{t}\right)-\left\|C_{\phi_{t}}\right\|^{2}
$$

is a continuous, real-valued function on the interval $(0,1)$. Suppose, for the sake of argument, that $\psi_{t}\left(\omega_{t}\right) \geq\left\|C_{\phi_{t}}\right\|^{2}$ for some $t$; in other words, $M(t) \geq 0$. We have already shown that $M(t)<0$ for values of $t$ close to 1 . Consequently there must be some $t_{0}$ in $(0,1)$ for which $M\left(t_{0}\right)=0$, or

$$
\psi_{t_{0}}\left(\omega_{t_{0}}\right)=\left\|C_{\phi_{t_{0}}}\right\|^{2} \text {. }
$$

Since the essential norm of $C_{\phi_{t_{0}}}$ is zero, we may apply (2.5) to obtain a function $g \in H^{2}$, vanishing nowhere on $\mathbb{U}$, with the property that $C_{\phi_{t_{0}}}^{*} C_{\phi_{t_{0}}} g=\left\|C_{\phi_{t_{0}}}\right\|^{2} g$. Now apply (2.8) with $g=f$; since $\omega_{t_{0}}$ is not a singularity for $\psi_{t_{0}}$ or $\chi_{t_{0}}$, we may substitute $\omega_{t_{0}}$ for $z$. Using the fact that $\tau_{t_{0}}\left(\omega_{t_{0}}\right)=\omega_{t_{0}}$, we obtain

$$
\left\|C_{\phi_{t_{0}}}\right\|^{2} g\left(\omega_{t_{0}}\right)=\psi_{t_{0}}\left(\omega_{t_{0}}\right) g\left(\omega_{t_{0}}\right)+\chi_{t_{0}}\left(\omega_{t_{0}}\right)\left\|C_{\phi_{t_{0}}}\right\|^{2} g(0) \text {. }
$$

Dividing both sides of the equation by $\psi_{t_{0}}\left(\omega_{t_{0}}\right)=\left\|C_{\phi_{t_{0}}}\right\|^{2}$, we see that

$$
g\left(\omega_{t_{0}}\right)=g\left(\omega_{t_{0}}\right)+\chi_{t_{0}}\left(\omega_{t_{0}}\right) g(0) .
$$

Since $\chi_{t_{0}}$ and $g$ are both non-vanishing functions, we arrive at a contradiction. In other words, $M(t)$ is negative for every $t$ in $(0,1]$, which proves our claim.

Lemma 4.2. Suppose that $\phi=\phi_{r, s}$ for some $s \in(0,1)$ and $r \in(-1,0)$, where $\phi_{r, s}$ is given by (1.31). Let $t \in(0,1]$, and let $\chi_{t}, \psi_{t}$, and $\tau_{t}$ be the usual auxiliary maps for $\phi_{t}$. Then for each $k \geq 0$,

$$
\chi_{t}\left(\tau_{t}^{[k]}\left(\phi_{t}(0)\right)\right) \prod_{m=0}^{k-1} \psi_{t}\left(\tau_{t}^{[m]}\left(\phi_{t}(0)\right)\right)>0 .
$$

Proof. We have seen that the auxiliary mappings $\chi$ and $\psi$ corresponding to $\phi$ are positive on the interval $(0,1]$, so that the same is true of $\chi_{t}$ and $\psi_{t}$ since $\chi_{t}(z)=\chi(t z)$ and $\psi_{t}(z)=\psi(t z)$.

We claim that $\tau_{t}$ is also positive on $(0,1]$. To prove the claim, it suffices to show that $\tau(x)$ is positive for $0<x \leq 1$ since $\tau_{t}(x)=t \tau(t x)$. We will show that $\tau$ is positive on the closed interval $[0,1]$. Note that

$$
\tau(x)=\frac{(r-1+2 s) x-r+s r+1-s}{(r-s r-1+s) x+1-r+2 s r} .
$$

Observe that $\tau(0)=(1-r)(1-s) /(1-r+2 s r)$ lies in the interval $(0,1)$ and that the unique singularity of $\tau$ is the reciprocal of $\tau(0)$. Thus $\tau$ is continuous and real-valued on $[0,1]$. Because $\tau(0)$ is positive, $\tau$ will be positive on $[0,1]$ if its zero,

$$
x_{0}:=\frac{(1-r)(1-s)}{1-r-2 s},
$$

lies outside of $[0,1]$. Note that the numerator of the fraction representing $x_{0}$ is positive. If $1-r-2 s<0$, then $x_{0}$ is negative and outside $[0,1]$, as desired. If $1-r-2 s=0$, then $\tau$ has no zero. Suppose that $1-r-2 s>0$; then an easy computation based on the negativity of $r$ shows that $x_{0}>1$, which completes our argument that $\tau$ is positive on $[0,1]$.

Because $\phi_{t}(0)=t(1-s) /(1+s r)>0$, the positivity of $\tau_{t}, \chi_{t}$, and $\psi_{t}$ on $(0,1]$ yields the inequality of the lemma. 
Theorem 4.3. Suppose that $\phi=\phi_{r, s}$ for some $s \in(0,1)$ and $r \in(-1,0)$, where $\phi_{r, s}$ is given by (1.3). Let $t \in(0,1)$, and let $\chi_{t}, \psi_{t}$, and $\tau_{t}$ be the usual auxiliary maps for $\phi_{t}$. Then $\lambda:=\left\|C_{\phi_{t}}\right\|^{2}$ is the unique positive number satisfying

$$
\sum_{k=0}^{\infty} \chi_{t}\left(\tau_{t}^{[k]}(\phi(0))\right)\left[\prod_{m=0}^{k-1} \psi_{t}\left(\tau_{t}^{[m]}\left(\phi_{t}(0)\right)\right)\right]\left(\frac{1}{\lambda}\right)^{k+1}=1 .
$$

Proof. Because $\left\|C_{\phi_{t}}\right\|>\left\|C_{\phi_{t}}\right\|_{e}=0$, (2.10) holds with $f$ representing an eigenvector for $C_{\phi_{t}}^{*} C_{\phi_{t}}$ associated with the eigenvalue $\lambda$ (where $f$ does not vanish on $\mathbb{U}$ ). Just as in the proof of Theorem 3.5. we divide both sides of (2.10) by $\lambda^{j+1}$ and take the limit as $j \rightarrow \infty$ to see that $\lambda$ must be a solution of (4.1). (Here Lemma 4.1 is needed.) Lemma 4.2 shows that the series coefficients of (4.1) are positive; thus there is only one positive solution of (4.1), which completes the proof.

Remarks. (a) Theorem 4.3 is also valid when $t=1$ (so that $C_{\phi_{t}}$ is not compact): for a proof, combine Theorem [3.7 Theorem [3.5] and Lemma 4.2

(b) The result of Theorem 4.3 also holds if we take $\phi(z)=\phi_{\alpha}(z)=(\alpha-1) /(\alpha-z)$ for $\alpha>1$. Indeed, when $\alpha>2$, recall that $\phi_{\alpha}=\phi_{r, s}$ for $r=-1 /(\alpha-1)$ and $s=1 /(\alpha-1)$. It is not difficult to handle the case where $1<\alpha \leq 2$. For $t$ in $(0,1]$, we can easily show that every point $\omega_{t}$ lies in the interval $(0,1]$. Observe that the auxiliary function

$$
\psi(z)=\frac{(\alpha-1) z}{\alpha-(\alpha-1) z}
$$

has the property that $0<\psi(x) \leq \alpha-1 \leq 1$ for $x$ in $(0,1]$; thus $0<\psi_{t}\left(\omega_{t}\right) \leq 1$. Because $\phi_{t}(0) \neq 0$, our general norm estimate (2.2) shows that $\left\|C_{\phi_{t}}\right\|>1$; hence the result of Lemma 4.1 follows immediately. Observe that $\chi_{t}=\chi \equiv 1$; we can easily show that the map $\tau$ is positive on $(0,1]$, from which we deduce the result of Lemma 4.2. Therefore Theorem 4.3 is also valid when $\phi=\phi_{\alpha}$ for some $\alpha>1$.

For each positive integer $n$, let

$$
p_{n}(\lambda)=\sum_{k=0}^{n} \chi_{t}\left(\tau_{t}^{[k]}\left(\phi_{t}(0)\right)\right)\left[\prod_{m=0}^{k-1} \psi_{t}\left(\tau_{t}^{[m]}\left(\phi_{t}(0)\right)\right)\right]\left(\frac{1}{\lambda}\right)^{k+1},
$$

where $\psi_{t}, \tau_{t}$, and $\chi_{t}$ are related to $\phi_{t}$ as in Theorem 4.3. For $n \geq 1$, let $m_{n}$ be the largest value of $\lambda$ for which $p_{n}(\lambda)=1$. Because the series coefficients in (4.1), which correspond to the coefficients of $p_{n}$, are all positive, it is easy to see that the sequence $\left(m_{n}\right)$ must increase with $n$ to $\left\|C_{\phi_{t}}\right\|^{2}$. We will use this fact for a sample calculation, after presenting a more explicit version of (4.1) that corresponds to $\phi$ of the form $\phi(z)=b /(c z+d)$.

We seek to compute the norm of $C_{\phi}$, where $\phi(z)=b /(c z+d)$ and $\|\phi\|_{\infty}<1$ (so that $C_{\phi}$ is compact). Note that if $b=0$, so that $\phi(0)=0$, then $\left\|C_{\phi}\right\|=1$; thus, for the remainder of the paper, we will consider $b$ to be non-zero. Setting $c_{1}=c / b$ and $d_{1}=d / b$, we may write $\phi(z)=1 /\left(d_{1}+c_{1} z\right)$. Let $\zeta$ be the point on the unit circle at which $\phi$ assumes its maximum modulus, so that $\zeta=-e^{i \arg \left(d_{1}\right)-i \arg \left(c_{1}\right)}$, and let $\eta=e^{i \arg \left(d_{1}\right)}$. Because $C_{\zeta z}$ and $C_{\eta z}$ are unitary, the norm of $C_{\phi}$ equals the norm of $C_{\eta \phi(\zeta(z))}$. Observe that

$$
\eta \phi(\zeta(z))=\frac{1}{\left|d_{1}\right|-\left|c_{1}\right| z}=\frac{\left|1 / c_{1}\right|}{\left|d_{1} / c_{1}\right|-z} .
$$


Thus, without loss of generality, we compute the norm of $C_{\phi}$ assuming that $\phi$ has the form

$$
\phi(z)=\frac{b}{d-z}, \text { where } b, d>0 \text { and } b<d-1,
$$

the strictness of the final inequality ensuring that $C_{\phi}$ is compact.

In interpreting sums in the following proposition, the reader should follow the conventions that $\sum_{k=1}^{-1}=-\sum_{k=-1}^{1}$ and $\sum_{k=1}^{0}=0$.

Proposition 4.4. Suppose that $\phi(z)=b /(d-z)$, where $0<b<d-1$, and that $\lambda=\left\|C_{\phi}\right\|^{2}$. Then $\lambda$ is the unique positive number satisfying

$$
\sum_{k=0}^{\infty} a_{k}\left(\frac{1}{\lambda}\right)^{k+1}=1,
$$

where for $k \geq 0$,

$$
a_{k}=\frac{b^{2 k}}{d^{2 k}+(-1)^{k} b^{2 k}+(-1)^{k} \sum_{m=1}^{k-1}(-1)^{m} d^{2 m} \sum_{j=0}^{k-m}\left(\begin{array}{c}
m+j \\
j
\end{array}\right)\left(\begin{array}{c}
k-1-j \\
k-m-j
\end{array}\right) b^{2 j}} .
$$

Proof. First observe that $\phi$ may be written in the form $\phi=t \phi_{\alpha}$, where $t=b /$ $(d-1)<1$ and $\alpha=d$. Also observe that $\chi \equiv 1$ for $\phi$. Thus, by remark (b) following Theorem 4.3 we need only prove that, for $k \geq 0$, the formula for $a_{k}$ in the statement of the proposition is equal to

$$
\prod_{m=0}^{k-1} \psi\left(\tau^{[m]}(\phi(0))\right)
$$

where $\tau(z)=\frac{b(b z-d)}{b d z-d^{2}+1}, \psi(z)=\frac{b z}{d-b z}$, and $\phi(0)=\frac{b}{d}$. This may be verified via a tedious inductive argument, which we omit.

Equation (4.3) simplifies considerably when $b=1$; in fact, the equation reduces to

$$
\sum_{k=0}^{\infty} \frac{(1 / \lambda)^{k+1}}{U(2 k, d / 2)}=1,
$$

where $U(j, \cdot)$ is the $j$-th Chebyshev polynomial of the second kind. In particular, $\lambda=\left\|C_{1 /(3-z)}\right\|^{2}$ is the the unique positive solution to

$$
\sum_{k=0}^{\infty} \frac{(1 / \lambda)^{k+1}}{F[4 k+2]}=1,
$$

where $F[j]$ represents the $j$-th Fibonacci number. As before, for a positive integer $n$, let $m_{n}$ denote the maximum solution of $\sum_{k=0}^{n} \frac{(1 / \lambda)^{k+1}}{F[4 k+2]}=1$. Then

$$
\begin{aligned}
& m_{1} \approx 1.11237243569, \quad m_{5} \approx 1.12731235144, \\
& m_{10} \approx 1.12731643238, \text { and } m_{20} \approx 1.12731643253 \text {. }
\end{aligned}
$$

Thus we assert that $\left\|C_{1 /(3-z)}\right\|^{2}$ is approximately 1.1273164 . 
Final remarks. (a) In the proof of Lemma 4.1, the fact that $\left\|C_{\phi}\right\|_{e}<\left\|C_{\phi}\right\|$ was of crucial importance. Indeed, to explain the behavior of the example presented at the beginning of this section, we observe that

$$
\frac{4 z+4}{z+12}=(8 / 13) \phi_{r, s}(z)
$$

where $s=11 / 26$ and $r=4 / 26$. For these values of $r$ and $s$, let $\phi=\phi_{r, s}$. For $t$ in $[8 / 13,1]$, we can verify directly that the values $\psi_{t}\left(\omega_{t}\right)$ are positive and vary continuously with $t$. Therefore the problematic nature of this example may be attributed in part to the fact that $C_{\phi}^{*}$ is subnormal, which implies that $\left\|C_{\phi}\right\|_{e}=$ $\left\|C_{\phi}\right\|$.

(b) Our methods will yield norms of certain compact linear-fractional composition operators which do not satisfy the hypotheses of Theorem 4.3 and which do not have the form $\phi(z)=b /(d-z)$. For example, it is possible to show that Lemma 4.1, Lemma 4.2 and Theorem 4.3 are all valid when $\phi=\phi_{r, s}$, where $\phi_{r, s}$ is given by (1.3) but where $s>1$ and $r<-1$. (Observe that the maps $\phi_{\alpha}$, where $1<\alpha<2$, belong to this family, with $\phi_{\alpha}=\phi_{r, s}$ for $r=-1 /(\alpha-1)$ and $s=1 /(\alpha-1)$.) These relatives of Cowen-Kriete mappings induce composition operators with essential norm less than 1 , so that the crucial inequality $\left\|C_{\phi}\right\|_{e}<\left\|C_{\phi}\right\|$ needed to obtain Lemma 4.1 holds simply because $\phi(0) \neq 0$.

\section{REFERENCES}

[1] M. Appel, P. Bourdon, and J. Thrall, Norms of composition operators on the Hardy space, Experiment. Math. 5 (1996), 111-117. MR 97h:47022

[2] P. Avramidou and F. Jafari, On norms of composition operators on Hardy spaces, Contemp. Math. 232, Amer. Math. Soc., Providence, 1999. MR 2000b:47066

[3] A. Baker, Transcendental Number Theory, Cambridge University Press, New York, 1975. MR 54:10163

[4] P. S. Bourdon and J. H. Shapiro, Cyclic Phenomena for Composition Operators, Memoirs Amer. Math. Soc. \#596, January 1997. MR 97h:47023

[5] P. S. Bourdon and D. Q. Retsek, Reproducing kernels and norms of composition operators, Acta Sci. Math. (Szeged) 67 (2001), 387-394. MR 2002b:47043

[6] P. S. Bourdon, D. Levi, S. Narayan, and J. H. Shapiro, Which linear-fractional composition operators are essentially normal?, J. Math. Anal. Appl. 280 (2003), 30-53. MR 2003m:47042

[7] C. C. Cowen, Composition operators on $H^{2}$, J. Operator Theory 9 (1983), 77-106. MR 84d:47038

[8] C. C. Cowen, Linear fractional composition operators, Integral Equations Operator Theory 11 (1988), 151-160. MR 89b:47044

[9] C. C. Cowen and T. L. Kriete, Subnormality and composition operators on $H^{2}$, J. Funct. Anal. 81 (1988), 298-319. MR 90c:47055

[10] C. C. Cowen and B. D. MacCluer, Composition Operators on Spaces of Analytic Functions, CRC Press, Boca Raton, 1995. MR 97i:47056

[11] C. C. Cowen and B. D. MacCluer, Some problems on composition operators, Contemporary Mathematics 213 (1998), 17-25. MR 99d:47029

[12] K. W. Dennis, Co-hyponormality of composition operators on the Hardy space, Acta Sci. Math. (Szeged) 68 (2002), 401-411. MR 2003f:47039

[13] P. L. Duren, Theory of $H^{p}$ Spaces, Academic Press, New York, 1970. MR 42:3552

[14] C. Hammond, On the norm of a composition operator with linear fractional symbol, Acta Sci. Math. (Szeged), to appear.

[15] E. A. Nordgren, Composition operators, Canadian J. Math. 20 (1968), 442-449. MR 36:6961

[16] D. B. Pokorny and J. E. Shapiro, Continuity of the norm of a composition operator, Integral Equations Operator Theory 45 (2003), 351-358.

[17] D. Q. Retsek, The Kernel Supremum Property and Norms of Composition Operators, Thesis, Washington University, 2001. 
[18] W. Rudin, Real and Complex Analysis, 3rd ed., McGraw-Hill, New York, 1987.

[19] H. Sadraoui, Hyponormality of Toeplitz and Composition Operators, Thesis, Purdue University, 1992.

[20] J. H. Shapiro, The essential norm of a composition operator, Annals of Math. 125 (1987), 375-404. MR 88c: 47058

[21] J. H. Shapiro, Composition Operators and Classical Function Theory, Springer-Verlag, New York, 1993. MR 94k:47049

Department of Mathematics, Washington and Lee University, Lexington, Virginia 24450

E-mail address: pbourdon@wlu.edu

Department of Mathematics, Washington and Lee University, Lexington, Virginia 24450

E-mail address: frye@wlu.edu

Department of Mathematics, University of Virginia, Charlottesville, Virginia 22904

E-mail address: cnh5u@virginia.edu

Current address: Department of Mathematics and Computer Science, Connecticut College, New London, Connecticut 06320

E-mail address: cnham@conncoll.edu

Department of Mathematics, Washington and Lee University, Lexington, Virginia 24450

E-mail address: spoffordc@wlu.edu

Current address: Department of Mathematics, University of Virginia, Charlottesville, Virginia 22904 